Submillimetre cosmology

Editors : will be set by the publisher

EAS Publications Series, Vol. ?, 2018

\title{
SUBMILLIMETRE COSMOLOGY AT HIGH ANGULAR RESOLUTION
}

\author{
Thomas R. Greve ${ }^{1}$
}

\begin{abstract}
Over the last decade observations at submillimetre (submm) and millimetre $(\mathrm{mm})$ wavelengths, with their unique ability to trace molecular gas and dust, have attained a central role in our exploration of galaxies at all redshifts. Due to the limited sensitivities and angular resolutions of current submm/mm telescopes, however, only the most luminous objects have been uncovered at high redshifts, with interferometric follow-up observations succeeding in resolving the dust and gas reservoirs in only a handful of cases. The coming years will witness a drastic improvement in the current situation, thanks to the arrival of a new suite of powerful submm observatories (single-dish and interferometers) with an order of magnitude improvement in sensitivity and resolution. In this overview I outline a few of what I expect to be the major advances in the field of galaxy formation and evolution that these new ground-breaking facilities will facilitate.
\end{abstract}

\section{Introduction}

Key to understanding the origins of galaxies and their supermassive black holes (SMBHs) is that benchmark period of cosmic history called the epoch of reionization (EoR) in which the radiation fields from the first stars and accreting black holes reionized the surrounding gas - an epoch of time that lasted from a couple of hundred million years to about one billion years after the Big Bang. While the first glimpses into the EoR have now been made owing to the discovery of a handful of quasars, Lyman-alpha emitters (LAEs) and gamma-ray bursts (GRBs) at $z \gtrsim 6$ (e.g. Fan et al. 2003; Kashikawa et al. 2006; Kawai et al. 2006), including the recently discovered GRB at $z \sim 8.3$ (corresponding to about 625 million years after the Big Bang - Tanvir et al. 2009), it remains terra incognita and a top priority of extragalactic astronomy in the years to come.

\footnotetext{
${ }^{1}$ Max-Planck Institute for Astronomie, Königstuhl 17, D-69117 Heidelberg, Germany
} 
Submillimetre cosmology

Another major challenge for extragalactic astronomy will be to make sense of the many different types of galaxies found at $z \lesssim 6$ in the context of galaxy evolution after the EoR. It is during this $\sim 12.7$ Gyr epoch - i.e. from the first one billion years to the present day - that the bulk growth and evolution of galaxies and SMBHs took place. High-resolution radio/near-IR/submm observations have shown that accurate measurements of the sizes, morphologies, and kinematics of the gas and stars in high- $z$ galaxies are crucial steps in delineating their evolutionary paths and linking them to galaxy populations at later cosmic epoch. Yet our understanding of the 'evolutionary tree' of galaxies is still rudimentary, with even seemingly simple questions such as 'how do galaxies attain their gas?', and 'what is the co-evolution of SMBHs and galaxies?' lacking definite answers.

While answering these questions will require a 'pan-chromatic' effort, I shall here focus on the unique contributions we can expect from the next generation of large submm observatories such as a European $25 \mathrm{~m}$ aperture submm telescope at Dome C, the Cornell Caltech Atacama Telescope (CCAT), the Atacama Large Millimeter Array (ALMA), and the Northern Extended Millimeter Array (NOEMA). The dramatic improvements in sensitivity, angular resolution, frequency coverage, and calibration capabilities of these facilities represent a quantum leap in our ability to map the gas and dust reservoirs in galaxies at all cosmic epochs, heralding in a new golden era in extragalactic astronomy.

\section{The first galaxies - peering into the epoch of reionization}

The high cosmic neutral gas fraction $\left(f_{\mathrm{HI}} \gtrsim 10^{-3}\right)$ at $z \gtrsim 6-$ inferred from the observed Gunn-Peterson absorption troughs towards high- $z$ QSOs (e.g. Fan et al. 2003 ) - implies that only observations longward of $\sim 0.9 \mu \mathrm{m}$ (and X-rays) are able to peer into the EoR.

Continuum observations probing the thermal dust emission at submm/mm wavelengths are particular powerful for this endeavour, given their unique negative $k$-correction which effectively offsets the cosmic dimming out to redshifts of $z \sim 10$ (Blain \& Longair 1993). The submm detection of the $z=6.42$ QSO SDSS J1148+5251 (Walter et al. 2004) demonstrated the presence of a large amount of dust $\left(\sim 10^{9} M_{\odot}\right)$, pointing to rapid dust production mechanisms at these early epochs. Type II supernovae (SNe) has been suggested as an important source of dust (Dunne et al. 2003), although according to models the contribution from AGB stars may also be substantial (Valiante et al. 2009). ALMA will probe $\gtrsim 10 \times$ (Fig. 1) further down the IR luminosity function at these redshifts than what is currently possible, detecting the rest-frame FIR/submm emission from 'normal' low-luminosity $\left(\sim 10^{10} L_{\odot}\right)$ galaxies, e.g. LAEs which models predict will be sufficiently submm bright $\left(S_{850 \mu \mathrm{m}} \sim 0.1 \mathrm{mJy}-\right.$ Dayal et al. 2009$)$. The superior continuum sensitivity of ALMA over a broad $\mathrm{mm} / \mathrm{submm}$ range $(84-950 \mathrm{GHz})$ combined with its remarkable angular resolution $\left(\sim 0.1^{\prime \prime}\right.$, corresponding to $\sim 0.6 \mathrm{kpc}$ at $z \sim 6$ ) will allow us to spatially resolve the dust emission, disentangle hot/cold dust components, determine accurate dust masses for large samples of $z>6$ galaxies, and ultimately reveal the dust production processes and 

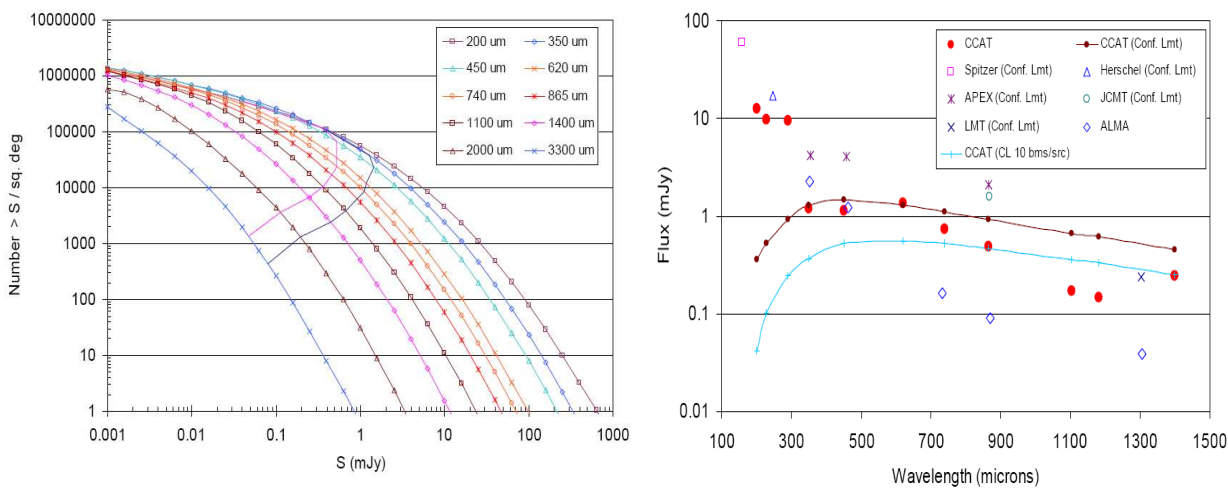

Fig. 1. Left: Models of the submm number counts at different wavelengths (Blain et al. 1999, 2002). The crossing lines show 30 (lower) and 10 (upper) beams/source confusion limits for a 25m single-dish telescope. Right: Continuum point source sensitivities (5- $\sigma$ ) after 1 hr integration vs. wavelength for CCAT (red circles) and ALMA (blue diamonds), along with confusion limits (30 beams/source) for a number of other submm/mm facilities. Adapted from the CCAT Science Case (v1.3).

time-scales in the early Universe.

Turning to the prospects of spectral line studies of galaxies within the EoR: the detections of low- to mid- $J$ CO lines in a handful of $z \sim 4-6$ QSOs by Weiß et al. (2006) led them to predict (based on extrapolation of models) that a) the $J \gtrsim 7$ lines are not significantly excited, and b) if similar excitation conditions prevail in $z \gtrsim 7$ objects, their CO detection-potential with ALMA is slim (due to its low-frequency cut-off at $84 \mathrm{GHz}$, which means only $J>7$ lines are observable at $z>7$ ). In the local Universe the nuclei of starburst and seyfert galaxies are found to have much higher $\mathrm{CO}$ line ratios (i.e. excitation conditions) than the global galaxy average (e.g. Papadopoulos \& Seaquist 2000). Since few of the high$z$ QSOs have been robustly resolved in $\mathrm{CO}$, it is possible that the same 'aperture' effects are at play here (and affecting the modeling), with the high- $J$ lines being excited in the nuclear/starburst regions only. The sensitivity and resolution of ALMA (and NOEMA) should be able to spatially isolate this nuclear high- $J$ CO emission from the galaxy regions further out, allowing for a more accurate picture of the ISM conditions in the first QSOs.

While QSOs are likely to harbor the most extreme excitation conditions possible, more normal high- $z$ galaxies are likely to have quiescent, possibly Milky Way-type, ISM conditions. Due to selection effects (bias towards luminous, midto high- $J$ CO lines at high $z$ ), only a few examples of such galaxies have been found at $z>1$ (Papadopoulos \& Ivison 2002; Greve et al. 2003; Dannerbauer et al. 2009), and as first pointed out by Papadopoulos \& Ivison (2002), galaxies with such ISM conditions at $z \gtrsim 7$ would be extremely difficult to detect in CO with ALMA. The recent detections of the [CII] $158 \mu$ m fine-structure line towards a handful of $z>4$ QSO (Maiolino et al. 2005, 2009; Iono et al. 2006; Walter 
Submillimetre cosmology

et al. 2009) have raised hopes for a more feasible way to study $z \gtrsim 7$ objects in the future. Not only is the [CII] line observable with ALMA at these redshifts, but in SDSS J1148+5251 the line is $\sim 5 \times$ brighter than the strongest CO line $(J=6-5)$. Furthermore, [CII] not only remains luminous in metal-poor systems (where CO is photo-dissociated by UV photons), which would favor its detection in low-mass systems, but it is also not exclusively tied to star-forming sites; in fact the cold and warm neutral medium as well as ionized gas (H II regions) can have significant [CII] emission contributions (Madden et al. 1997), in which case sensitive, high-resolution observations could reveal 'hidden' reservoirs of gas that CO would not pick up.

\section{Galaxy evolution after the first one billion years}

\subsection{The dust-obscured Universe}

The first extragalactic submm $(850-\mu \mathrm{m})$ surveys with SCUBA/JCMT (Barger et al. 1998; Hughes et al. 1998) showed that a significant fraction of the cosmic star formation at $z \sim 1-3$ occurred in heavily dust-obscured galaxies, so-called submm-selected galaxies (SMGs - Blain et al. 2002), that had been missed by UV/optical surveys. Such surveys, with their coarse resolution (FWHM $\sim 15^{\prime \prime}$ ) and confusion limit $(\sim 2 \mathrm{mJy})$, only select the most luminous $\left(\sim 10^{13} L_{\odot}\right)$ objects of the population, although surveys using galaxy clusters as 'cosmic magnification glasses' have been able go somewhat beyond the formal confusion limit of blankfield surveys (reaching depths of $\sim 1 \mathrm{mJy}$ at $850-\mu \mathrm{m}-$ Smail et al. 2002; Chapman et al. 2003; Knudsen et al. 2008).

However, the next generation of single-dish submm telescopes - i.e. 20-30 m single-dish antennas located at Chajnantor and/or Dome C - equipped with largeformat filled-array cameras capable of effectively operating at $\sim 200-850 \mu \mathrm{m}$ would have the sufficient angular resolution $\left(\sim 2-4^{\prime \prime}\right)$ and field of view $\left(\sim 10^{\prime}-20^{\prime}\right)$ to map several square degrees of sky down to depths of $\lesssim 0.5 \mathrm{mJy}$ (Fig. 1), thereby a) uncover tens of thousands of sub-mJy SMGs, b) sample the entire 'dust' luminosity function, and c) resolve the entire FIR/submm background. The relatively small beam sizes of such large single-dish telescopes, combined with observations in multiple submm bands would allow for robust counterpart identifications and photometric redshift estimates (Hughes et al. 2002; Aretxaga et al. 2003). Interferometers with their small fields of view are unlikely useful blank-field survey facilities. For example, given the primary beam of ALMA $\left(\sim 9^{\prime \prime}-56^{\prime \prime}\right.$ from Band 9 to 3$)$, a single, deep $(\sigma \sim 3 \times 10 \mu \mathrm{Jy})$ 'blank' pointing is expected to yield no more than 1-2 sources - making it very time-consuming to robustly constrain the faint number counts. Instead, the main strength of submm $/ \mathrm{mm}$ interferometers will be as follow-up facilities, providing extremely high-resolution and high-fidelity continuum imaging of the dust reservoirs in pre-selected galaxies.

The FIR/submm spectral energy distributions (SEDs) of high- $z$ galaxies are usually inferred from fits of simple, optically thin dust emission models to a few $(<4)$ global, i.e. unresolved, photometric data points. However, it has been sug- 
gested that the dust in local Ultra Luminous Infra-Red Galaxies $\left(L_{\mathrm{IR}} \geq 10^{12} L_{\odot}\right.$ - ULIRGs) could be optically thick at FIR/submm wavelengths (Condon et al. 1991; Solomon et al. 1997; Lisenfeld et al. 2003), in which case a revision of of dust mass and temperature estimates may be in store, as would the usefulness of FIR/submm line diagnostics. In SMGs where the gas and dust may be even more densely packed than in local examples (cf. Iono et al. 2008), optical depth effects may play an even bigger role. The problem of dust opacity will be addressed by future submm interferometers, by resolving the dust emission on sub-kpc scales within galaxies over a broad range of frequencies (Fig. 2). Furthermore, such dust maps would match the resolution of current radio observations, allowing for resolved FIR-radio correlation (Condon 1992) studies in galaxies out to $z \sim 3$ (the limit is set by the radio, not the submm, observations). This would shed important new light on the underlying physics of the correlation, e.g. its dependence on the the initial mass function and the magnetic field vs. CMB energy density ratio.

\subsection{Galaxy formation: major mergers or cold accretion?}

How did galaxies accrete their gas - was it primarily via one or two major mergers, interspersed with periods of quiescent evolution, or did they assemble and evolve through a prolonged, steady 'cold' accretion of gas? Observational evidence for both scenarios is found at high redshifts, yet the details of these two modes of galaxy formation remain unclear, as does their relative importance with redshift, galaxy/halo mass, and environment.

There is now strong observational evidence in favour of bright $\left(S_{850 \mu \mathrm{m}} \gtrsim 5 \mathrm{mJy}\right)$ SMGs being the progenitors of massive spheroids. Their extreme star formation rates $\left(\sim 1000 M_{\odot} \mathrm{yr}^{-1}\right)$ and their co-moving number density $\left(\sim 10^{-5} \mathrm{Mpc}^{-3}\right)$, which is similar to that of giant ellipticals at later epochs, both point in this direction. Moreover, interferometric $\mathrm{CO}$ observations have determined gas masses $\left(M_{\text {gas }} \sim 3 \times 10^{9} M_{\odot}\right)$, physical sizes $\left(R_{1 / 2} \sim 2 \mathrm{kpc}\right)$, line widths (FWHM $\sim$ $\left.700 \mathrm{~km} \mathrm{~s}^{-1}\right)$, dynamical masses $\left(M_{d y n} \sim 10^{11} M_{\odot}\right.$ within $\left.R_{1 / 2}\right)$ and gas fractions $\left(f_{\text {gas }} \sim 0.4\right)$, all of which strongly suggest that SMGs are gas-rich, highlydissipative major mergers in which the gas is rapidly funneled towards the center due to angular momentum loss via dissipation, igniting an intense 'maximal' starburst capable of producing a $2-3 m_{*}$ galaxy within a few hundred million years (Neri et al. 2003; Greve et al. 2005; Tacconi et al. 2006, 2008; Swinbank et al. 2006). The inferred central surface densities in SMGs, however, are $\sim 10 \times$ those observed in $z \sim 0$ spheroids (Bouché et al. 2007; Tacconi et al. 2008), which have led to the suggestion that $z \sim 2$ SMGs are the direct progenitors of the recently discovered population of extremely compact ( $R \sim 1 \mathrm{kpc}$ ), very dense, 'dead' galaxies at $z \sim 1.5$ (Trujillo et al. 2006; Zirm et al. 2007; Toft et al. 2007). The latter, in order to reach the larger half-light sizes and lower central densities of today's early-type galaxies, will have had to undergo 'dry' accretion, i.e. mergers where little or no gas is involved (van Dokkum 2005; Naab et al. 2007), or a combination of both (Burkert et al. 2008). The above evolutionary scenario is still very tenta- 

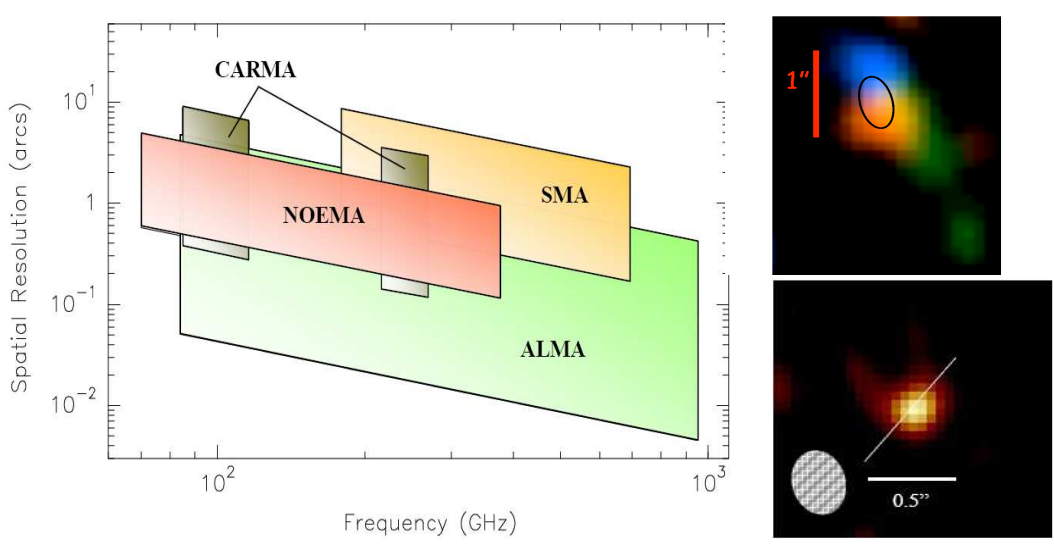

Fig. 2. Left: Angular resolution vs. observing frequency for current submm $/ \mathrm{mm}$ interferometers (SMA, CARMA) and future facilities (NOEMA, ALMA). Adopted from the NOEMA Phase-A study document. Right: High-resolution CO (3-2) (top) and (6-5) (bottom) maps of SMGs at $z=1.53$ (Bothwell in prep.) and $z=2.20$ (Tacconi et al. 2008), respectively. The CO (3-2) map is color coded by velocity.

tive, however, and in fact we note that far from all SMGs are compact, some have $\mathrm{CO}$ and radio morphologies extended up to $\sim 5-10 \mathrm{kpc}$ scales (Chapman et al. 2004; Bothwell et al. in prep.; Fig. 2).

Major mergers also seem to be associated with the most luminous high- $z$ radio galaxies (HzRGs) and optically selected QSOs, a third of which have been shown to be extremely IR-luminous $\left(L_{\mathrm{IR}} \simeq 10^{13} L_{\odot}-\right.$ Omont et al. 2003). Subsequent high-resolution interferometric submm observations of a subset of those have found massive $\left(\sim 10^{10} M_{\odot}\right)$, but often spatially and kinematically distinct, gas and dust components (Papadopoulos et al. 2000; Riechers et al. 2008). While this is indicative of an ongoing major 'wet' merger, the overall masses and luminosities involved are substantially larger than those of SMGs, giving the impression that we are dealing with a much more violent event, possibly a 'monolithic' collapse of a giant gas cloud which occurs in only the most highly mass-biased and rare peaks of the cosmic density field.

Observational evidence for galaxy growth via the steady accretion of gas flows and minor mergers has come from kinematically resolved $\mathrm{H} \alpha$ surveys of UV/optical selected galaxies at $z \sim 1-3$ using integral field units (e.g. Förster-Schreiber et al. 2006; Genzel et al. 2006). While these galaxies are as massive as SMGs $\left(M_{d y n} \sim 10^{11} M_{\odot}\right)$, their morphologies and kinematics differ fundamentally from SMGs in that they have disk-like shapes and show $(\mathrm{H} \alpha)$ velocity fields resembling that of disks, with some being dominated by rotation (circular velocities of $v_{c} \sim 250 \mathrm{~km} \mathrm{~s}^{-1}$ ) and others by turbulent motion (velocity dispersions of $\sigma \sim$ $50 \mathrm{~km} \mathrm{~s}^{-1}$ ). This picture is supported by recent interferometric $\mathrm{CO}$ observations towards a few of these galaxies (Daddi et al. 2008; Dannerbauer et al. 2009), which 
found (tentative) evidence for extended emission $\left(R_{1 / 2} \sim 4 \mathrm{kpc}\right)$ and rotation consistent with a disk. In addition, evidence of low-excitation gas (subthermal CO $3-2 / 1-0$ line ratio) was found, akin to the 'moderate' ISM conditions in local spirals and our own Galaxy. Furthermore, their average star formation efficiencies (as gauged by $L_{\mathrm{IR}} / L_{\mathrm{CO}}^{\prime}$ ) was found to be $4-5 \times$ below that of SMGs, but consistent with that of local spirals. Simulations suggest that the high degree of gas turbulence found in some of these high- $z$ disks $\left(v_{c} / \sigma \lesssim 3\right.$, compared to $v_{c} / \sigma \sim 10$ in local disks) may be driven by the kinetic energy gained by the gas as it streams inwards along filaments, in turn causing disk-instability and the subsequent breakup into giant clumps of gas, capable of forming stars efficiently (Dekel et al. 2009).

With ALMA and NOEMA it will be possible to very effectively target large samples of pre-selected high- $z$ galaxies across a broad range of redshifts, masses and selection criteria, and by means of a rich variety of ISM diagnostic molecular (e.g. $\mathrm{CO}, \mathrm{HCN}, \mathrm{HCO}^{+}$) and atomic lines (e.g. [C II] $158 \mu \mathrm{m}$ and [O I] 63 and $145 \mu \mathrm{m}$ ), accurately measure their (resolved) velocity fields, dynamical masses, gas fractions and morphologies. The deep understanding of the gas accretion processes in high- $z$ galaxies, and their evolutionary links to intermediate- and low- $z$ galaxy populations, is just one major result that will be gleaned from such studies. They will also provide a rather exhaustive account of the physical properties of the ISM (e.g. density and temperature regimes, heating and cooling mechanisms, UV field strength). Linking these to the distributed star formation (traced by dust emission) we can explore changes in the slope of the star formation law for different types of high- $z$ galaxies and different gas density tracers, and whether the slope goes from superlinear to linear once sub-kpc scales are probed (Bigiel et al. 2008).

\section{Supermassive black holes and their host galaxies}

\subsection{The origin of supermassive black holes}

Most galaxies in the present-day Universe harbour a SMBH in their centers (e.g. Kormendy et al. 1996, 1997), yet the origins of these black holes remain a mystery. In all likelihood, however, they grew from 'seed' black holes that formed very early in the Universe's history $(z>10)$. Intermediate-mass $\left(\sim 10^{2} M_{\odot}\right)$ BHs resulting from Pop III stars going supernova, may have been such 'seeds' for merger trees to SMBHs (e.g. Ohkubo et al. 2009). In an alternative scenario the monolithic gravitational collapse of primordial gas clouds led to the formation of 'seeds' with initial masses of $\sim 10^{5} M_{\odot}$ (Bromm \& Loeb 2003). The latter scenario might be favored as the progenitors of the $\sim 10^{9} M_{\odot}$ SMBHs found in $z \gtrsim 6$ QSOs (e.g. Becker et al. 2001), as it is difficult to make Pop III BHs grow so massive in only a few hundred million years even if they are accreting at super-Eddington rates. One may speculate, however, whether Pop III remnants provided the 'seeds' for the SMBHs in less massive, but more abundant, galaxies, and whether the SMBH growth in these galaxies occurred at a much more leisurely rate?

It was recently proposed that the high- $J$ rotational transitions of $\mathrm{CO}, \mathrm{HCN}$, 
$\mathrm{HCO}^{+}$as well as the $[\mathrm{C} \mathrm{II}]$ and $[\mathrm{O} \mathrm{I}]$ fine-structure lines, may be used as markers of distant SMBHs (Meijerink \& Spaans 2005, 2006). As a general result - valid over a broad swath of gas conditions - models predict that the high- $J(J>6)$ lines are much more excited in X-ray dominated regions (XDRs - Maloney et al. 1996) where the gas is exposed to an intense, hard X-ray radiation field $(E=1-100 \mathrm{keV})$ characteristic of an AGN, than in environments where the gas is being irradiated by FUV-photons $(E=6-13.6 \mathrm{keV})$ representative of the Photo-dominated regions (PDRs - Tielens \& Hollenbach 1985) found in OB star forming regions. The reason for this is the higher gas temperatures $\left(10^{2}-10^{3} \mathrm{~K}\right)$ in XDRs caused by fast, penetrative electrons produced via X-ray photo-ionization, compared to the much more modest heating in PDRs via photo-electric emission from dust grains and UV pumping of $\mathrm{H}_{2}$.

While this is a novel and untested technique, it is worth noticing that interferometric studies of $\mathrm{CO}, \mathrm{HCN}$ and $\mathrm{HCO}^{+}$emission towards a small number of local IR-luminous galaxies where the presence of a powerful AGN is known by some other means, found elevated $\mathrm{HCN}$ and $\mathrm{HCO}^{+}$1-0 line fluxes (Kohno et al. 2003; Imanishi et al. 2007), in qualitative agreement with the enhanced HCN abundance expected in a XDR-type chemistry (Lepp \& Dalgarno 1988). The high- $J$ lines, however, which are expected to be even stronger AGN vs. starburst discriminants, have so far not been investigated in local galaxies due to opaqueness of the atmosphere at $\gtrsim 400 \mathrm{GHz}$. ALMA will have the frequency coverage and sensitivity to detect the high- $J$ lines towards galaxies out to $z \gtrsim 10$ (Spaans \& Meijerink 2008). High angular resolution $\left(\lesssim 0.1^{\prime \prime}\right.$, corresponding to $\lesssim 500 \mathrm{pc}$ at $z \sim 10$ ) will be important for such AGN vs. starburst studies, since the high- $J$ CO emitting gas associated with a XDR/AGN is likely to be compact $(R \lesssim 500 \mathrm{pc})$, and blindly modeling the global line ratios is therefore prone to a 'contaminating' PDR contribution from distributed starforming gas.

\subsection{The early co-evolution of SMBHs and their host galaxies}

Optical spectroscopy of spheroids in the local Universe have established tight relations between SMBHs and the luminosities, masses and velocity dispersions of their host galaxies (Magorrian et al. 1998; Gebhardt et al. 2000), suggestive of a self-regulating, physical connection between SMBH growth and spheroid build-up. High-resolution maps of the gas morphology and kinematics in the central regions of high- $z$ galaxies will allow us to study AGN fueling mechanisms and feedback processes on sub-kpc scales, thereby playing an important role in probing the physics behind the above scaling relations and their evolution with redshift.

Optical/NIR measurements of spheroid masses $\left(M_{\mathrm{sph}}\right)$ at high- $z$ are difficult to come by, since the luminous AGN tends to 'drown out' any other light. Instead, some of the strongest $M_{\mathrm{sph}}$-constraints have come from dynamical mass estimates derived from high-resolution $\mathrm{CO}$ (or [CrI]) maps of IR-luminous $z>4$ QSOs (Walter et al. 2003, 2009; Riechers et al. 2008), leading to the first indications that luminous QSOs at these early epochs had $M_{\mathrm{BH}} / M_{\mathrm{sph}}$ ratios $\gtrsim 10 \times$ above the local relation $\left(M_{\mathrm{BH}} / M_{\mathrm{sph}} \simeq 1.4 \times 10^{-3}\right)$. CO observations of optically luminous 

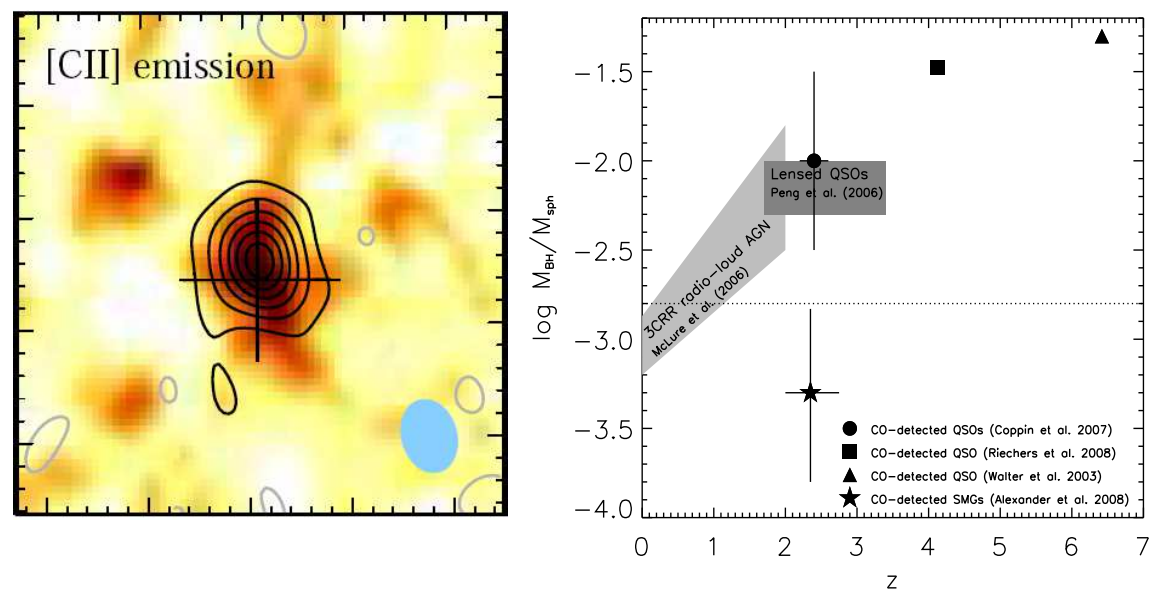

Fig. 3. Left: High-resolution ( $0.3^{\prime \prime}$ FWHM) maps of the [CII] (contours) and $\mathrm{CO}(3-$ 2) (color scale) emission toward the $z=6.42$ QSO J1148. Adapted from Walter et al. (2009). Right: Observational constraints from high-resolution CO studies on the the $\mathrm{SMBH}$-spheroid mass ratio for different types of galaxies at various redshifts. The dotted line represents the local ratio and no evolution. Adapted from Coppin et al. (2008).

$\left(M_{\mathrm{B}} \sim-28\right)$, submm-detected QSOs at $z \sim 2$ find $M_{\mathrm{BH}} / M_{\mathrm{sph}} \sim 9 \times 10^{-3}$ (Coppin et al. 2008), i.e. also significantly above the local ratio. The CO gas-dynamics of submm-detected, but optically less luminous $\left(M_{\mathrm{B}} \sim 25\right)$ QSOs at $z \sim 2$, however, suggest that they lie within the local relation given the uncertainties. Finally, high-resolution CO studies of SMGs (in conjunction with X-ray and optical/NIR observations) have shown that SMGs lie $\sim 3-5 \times$ below the local $M_{\mathrm{BH}}-M_{\mathrm{sph}}$ relation (Alexander et al. 2008). Thus, it appears that the $M_{\mathrm{BH}}-M_{\mathrm{sph}}$ relation evolves with redshift, and that it does so differently for different types of galaxies. In optically luminous QSOs, the build-up of the spheroid stellar mass lags the black hole growth, while in SMGs the situation appears to be reversed.

Substantial uncertainties are associated with the above findings, however, owing to the often poorly constrained CO (or [C II]) sizes and inclination angles. More worrying, though, for such potentially dynamically unsettled (non-virialized) systems, is the possibility that the gas might not always be centered around the black hole or even be a good probe of the dynamical mass. Examples of such AGN $/ \mathrm{H}_{2}$ configurations have recently been found at both high and low $z$ (Ivison et al. 2008; Riechers et al. 2008; Papadopoulos et al. 2008). ALMA, with its ability to spatially and kinematically resolve the gas reservoirs in distant galaxies on sub-kpc scales at high $S / N$, will explore these effects in great detail, allowing for more accurate spheroid and dynamical mass estimates in less luminous systems than is currently possible, thus facilitating a more straightforward comparison with local galaxies.

Estimating SMBH masses will remain challenging, however, and will initially at least be limited to QSOs/type-I AGN and SMGs where locally calibrated tech- 
niques for measuring black hole masses, such as reverberation mapping (Onken et al. 2004; Greene \& Ho 2005) and single epoch virial estimates (Vestergaard \& Peterson 2006), can be applied. With the advent of James Webb Space Telescope (JWST), however, there is hope that significantly improved SMBH mass estimates will be possible from direct imaging of the (atomic) gas and stellar kinematics (Ferrarese \& Ford 2005). Studying the (mass-)interrelationship of SMBHs and their host galaxies as a function of redshift and luminosity is likely to become an area of great synergy between ALMA and JWST. By contrasting the various SMBH-spheroid correlations to model predictions of their evolution (Di Matteo et al. 2005; Granato et al. 2004; Dotti et al. 2007) we will gain a much deeper insight into the underlying physics and the role SMBH and AGN-feedback plays in the overall scheme of galaxy formation and evolution.

Acknowledgments I'm grateful to the organizers and participants of the ARENA meeting for an interesting and stimulating meeting. I thank Matt Bothwell and Scott Chapman for providing me with a figure from Bothwell et al. in prep. I'm also thankful to F. Walter, C. Carilli, R. J. Ivison, P. P. Papadopoulos for useful comments.

\section{References}

Blain, A.W. et al. 2002, PhR, 369, 111

Bouché, N. et al. 2009, ApJ, 671, 303

Chapman, S.C. et al. 2004, ApJ, 614, 671

Coppin, K.E.K. et al. 2008, MNRAS, 389, 45

Daddi, E. et al. 2008, ApJ, 673, L21

Fan, X. et al. 2003, AJ, 125, 1649

Genzel, R. et al. 2006, Nature, 442, 786

Ivison, R.J. et al. 2008, MNRAS, 390, 1117

Kashikawa, N. et al. 2006, ApJ, 468, 7

Kawai, N. et al. 2006, Nature, 440, 184

Kormendy, J. et al. 1996, ApJ, 459, L57

Maiolino, R. et al. 2009, A\&A, 500, L1

Meijerink, R. \& Spaans, M. 2005, A\&A, 436, 397

Naab, T. et al. 2007, ApJ, 658, 710

Neri, R. et al. 2003, ApJ, 597, L113

Riechers, D.A. et al. 2008, ApJ, 686, L9

Solomon, P.M. et al. 1997, ApJ, 478, 144

Papadopoulos, P.P. \& Ivison, R.J. 2002, ApJ, 564, L9

Swinbank, A.M. et al. 2006, MNRAS, 371, 465

Smail, I. et al. 2002, MNRAS, 331, 495

Tacconi, L.J. et al. 2008, ApJ, 680, 246

Tanvir, N.R. et al. 2009, Nature, 461, 1254

Toft, S. et al. 2007, ApJ, 671, 285

Trujillo, I. et al. 2006, MNRAS, 373, L36

Walter, F. et al. 2009, Nature, 457, 699 